\title{
Chaos in collapsing Bose-condensed gas
}

\author{
Victo S. Filho, ${ }^{1}$ A. Gammal, ${ }^{1}$ T. Frederico, ${ }^{2}$ and Lauro Tomio ${ }^{1}$ \\ ${ }^{1}$ Instituto de Física Teórica, Universidade Estadual Paulista, 01405-900, São Paulo, Brazil \\ ${ }^{2}$ Departamento de Física, Instituto Tecnológico da Aeronáutica, CTA, 12228-900, São José dos Campos, Brazil
}

(Received 9 Feburary 2000; published 10 August 2000)

\begin{abstract}
We reinvestigate the dynamics of the grow and collapse of Bose-Einstein condensates in a system of trapped ultracold atoms with negative scattering lengths, and found a new behavior in the long time scale evolution: the number of atoms can go far beyond the static stability limit. The condensed state is described by the solution of the time-dependent nonlinear Schrödinger equation, in a model that includes atomic feeding and three-body dissipation. Our results for the model show that, by changing the feeding parameter and when a substantial depletion of the ground-state exists, a chaotic behavior is found. We consider a criterion proposed by Deissler and Kaneko [Phys. Lett. A 119, 397 (1987)] to diagnose spatiotemporal chaos.
\end{abstract}

PACS number(s): 03.75.Fi, 05.45.Mt, 32.80.Pj, 05.45.Pq

It has been shown numerically [1] and by experiments [2] that Bose-Einstein condensation (BEC) can occur in atomic traps even with attractive two-body interactions, as in ${ }^{7} \mathrm{Li}$. In this case, it was observed the occurrence of a critical maximum number of atoms $\left(N_{c}\right)$ in the ground state level; above such limit the condensate collapses under two body attraction. Recombination losses prevent the condensate to collapse to a point. Numerical simulations of this process were considered in Ref. [3], by studying the time evolution of the condensed wave function of atoms of ${ }^{7} \mathrm{Li}$ [4]. As noticed in Ref. [4], even considering the qualitative similar behavior of the theoretical simulation of Ref. [3] and their experimental measurements, there is relevant quantitative difference between the predictions with respect to the remaining number of atoms in the condensate. It is also reported in Ref. [4] that their observations could be "a first indicator of a complex dynamics accompanying BEC in a gas with attractive interactions." This strongly suggests to study the time evolution of BEC atoms for long periods, through the numerical solution of the corresponding time-dependent nonlinear Schrödinger equation (NLSE), as given in Ref. [3]. This equation includes two nonconservative (imaginary) terms: one, linear, related to the feeding of the condensate from the nonequilibrium thermal cloud, another, nonlinear and dissipative, corresponding to three-body recombination.

It is also well known that systems with complex dynamics can present chaotic behaviors for some appropriate range of parameters. In particular, we should note that the transition from a complex dynamics to chaos was previously considered in the time-dependent NLSE by other authors [5,6]. As shown in Ref. [6], for a definite set of parameters, small errors of the order of roundoff grow rapidly and saturate at values comparable in magnitude to the amplitude of the wave function itself, which lead to serious numerical instabilities of the solutions. Deissler and Kaneko, in Ref. [5], have also proposed a useful criterion to diagnose spatiotemporal chaos in NLSE, which relies on the determination of the time evolution of a function defined by the integral of the square modulus of the difference between wave functions with nearby initial conditions. The average slope of this function, when plotted as a function of time, gives the largest Lyapunov exponent.
The main purpose of the present paper is to analyze the model given in Ref. [3], and the consequences of the corresponding parametrization. Actually, it is very relevant to build a realistic model to describe the grow and collapse of the condensate for atomic systems with attractive two-body interactions, and Ref. [13] gives a very important contribution in this respect. The mean-field approximation was found to be a good approximation in the thermodynamical equilibrium, as observed in a quantum Monte Carlo calculation in Ref. [7]; however, the validity of this approximation to describe a physical system can be questionable in a complex dynamics scenario. In the present study, as it will be shown, for certain regime of the nonlinear parameters, the meanfield approximation can lead to a chaotic behavior. This is a new fact that should be considered in any improvements of the model.

In the present work, we use the criterions considered in Ref. [5] in order to verify numerically the onset of chaotic behavior of the solution of the time-dependent NLSE, which was considered in Ref. [3] for a trapped gas with attractive two-body interaction. In Ref. [8], the complex dynamics accompanying $\mathrm{BEC}$ of ${ }^{7} \mathrm{Li}$ atoms, was observed in the time evolution of the number of atoms in the condensate. It was verified the high sensibility of the numerical accuracy with the change of parameters, such that when a repulsive threebody interaction was considered, the numerical results were more stable for the condensate [8]. Later on, we verified that the numerical precision decreases very fast by increasing the modulus of the strength of an attractive three-body interaction. This preliminary result lead us to the suspicion of a possible chaotic behavior of the time-dependent NLSE with trapped atoms. In the next, we briefly describe the NLSE for trapped atoms considered in this letter, followed by the main results and conclusions.

We start our dynamical study by considering the NLSE corresponding to the one given in Ref. [3] for the trapped atoms with attractive two-body interaction. Two nonconservative terms were added to take into account, respectively, the decrease of the density due to three-body recombination (parametrized by $\xi$ ), and the feeding of the condensate from the nonequilibrium thermal cloud (parametrized by $\gamma$ ). The NLSE considered in this work is the mean-field approxima- 


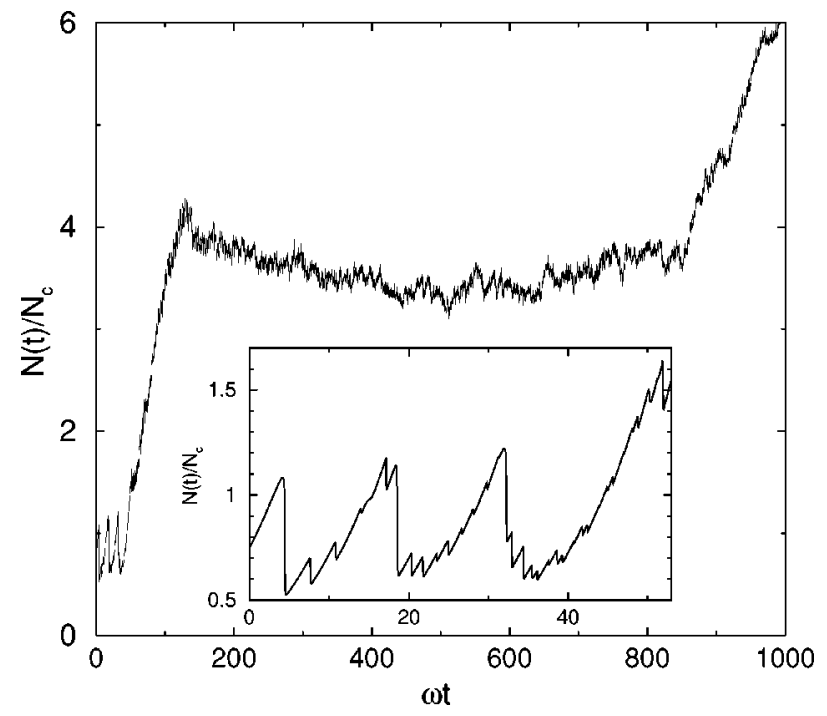

FIG. 1. Number of condensed atoms as a function of time for $\omega t \leqslant 1000$. The parameters are $\gamma=0.1$ and $\xi=0.001$. A doubling pattern is observed for $\omega t<50$, as shown in the inset. All quantities shown are dimensionless.

tion to the quantum many-body problem of a dilute gas in which the average interparticle distance is much larger than the absolute value of the scattering length. Also, the validity of such approximation is limited to wave-lengths much larger than the average interparticle distance.

In dimensionless units, as given in Eq. (2) of Ref. [8], the $s$-wave radial NLSE can be written as

$$
i \frac{\partial \Phi}{d \tau}=\left[-\frac{d^{2}}{d x^{2}}+\frac{1}{4} x^{2}-\frac{|\Phi|^{2}}{x^{2}}-2 i \xi \frac{|\Phi|^{4}}{x^{4}}+i \frac{\gamma}{2}\right] \Phi,
$$

where $x$ is related to the physical radius $\vec{r}$ by $x$ $\equiv \sqrt{2 m \omega / \hbar}|\vec{r}|, \quad \tau \equiv \omega t$ is the dimensionless time variable, with $\omega$ the frequency of the harmonic trap interaction. $\xi$ is the dissipation parameter, originated from three-body collisions, and $\gamma$ is a parameter related to the feeding of atoms from the thermal cloud (Ref. [3] gives an estimative for such parameters). $\Phi \equiv \Phi(x, \tau)$ is related to the physical wave function $\Psi(\vec{r}, t)$ by $\Phi(x, \tau) \equiv \sqrt{8 \pi N(t)|a|}|\vec{r}| \Psi(\vec{r}, t)$, where $N(t)$ is the number of atoms and $a$ is the two-body scattering length (here, assumed to be negative). Using these definitions, $\Psi(\vec{r}, t)$ is normalized to one and $\Phi(x, \tau)$ is normalized to the reduced number of atoms $n(\tau) \equiv 2 N(t)|a| \sqrt{2 m \omega / \hbar}$ :

$$
\int_{0}^{\infty} d x|\Phi(x, \tau)|^{2}=n(\tau)
$$

In order to obtain numerical solutions of Eq. (1), we applied the semi-implicit Crank-Nicolson (CN) algorithm as described, for instance, in Ref. [9] for nonlinear problems. We consider the same parameters given in Ref. [3] ( $\gamma=0.1$ and $\xi=0.001)$ and use the same initial condition they used for the number of atoms in the condensate $\left[N(t) / N_{c}\right.$ $\left.=n(\tau) / n_{c}=0.75\right]$. We should remark that, for the ${ }^{7} \mathrm{Li}$ condensed gas, the above dissipation parameter $\xi$ is about two to

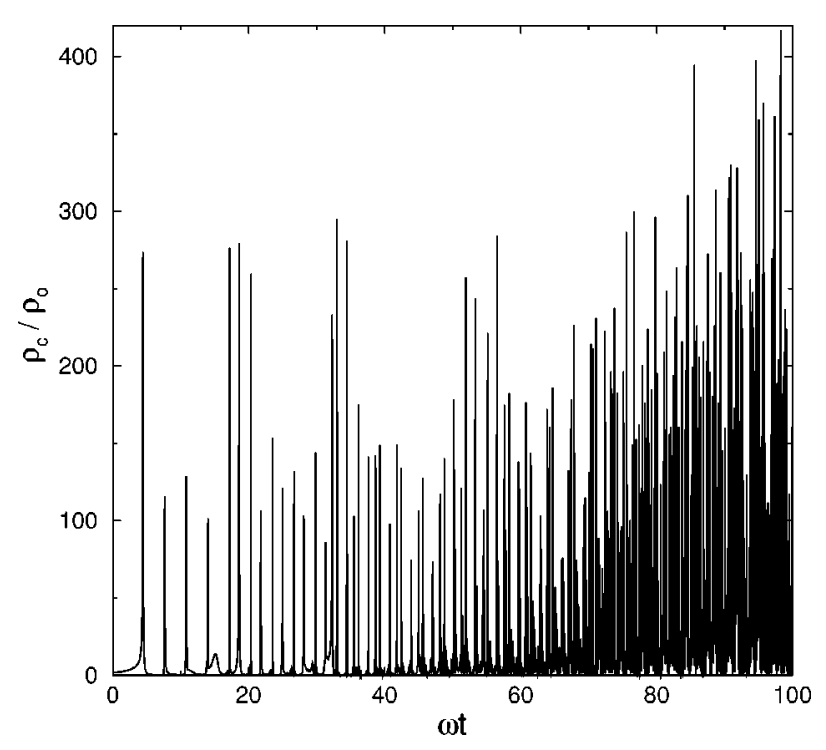

FIG. 2. The central density, $\rho_{c}(t) \equiv N|\Psi(0, t)|^{2}$, in dimensionless units, with $\rho_{0}$ defined by $\rho_{0} \equiv(1 / 4 \pi a)(m \omega / \hbar)$, is represented as a function of $\omega t$, for $\omega t<100$.

three orders of magnitude larger than the experimental one [10]. However, for our general study of Eq. (1) we use the parameters given in Ref. [3], in the perspective of application of the model to a more general atomic systems. We should note that, in principle, as discussed in Ref. [11], it is possible to alter experimentally the two-body interaction by means of light or induced magnetic field, and consequently affecting the three-body recombination rate. In respect to the feeding parameter $\gamma$, we allow a range of values up to the value used in Ref. [3]. The chaotic behavior of Eq. (1) is very sensible to the increasing of such parameter.

In Fig. 1 we show the evolution of the number of atoms for $\omega t \leqslant 1000$ (about 20 times larger than the maximum time considered in Fig. 1 of Ref. [3]). As already explained in Ref. [3], some dynamical collapses occur with frequency $\sim \omega$. Nevertheless a more careful analysis of such dynamical collapses show that the number of small peaks begins to double after each strong collapse, in a kind of fractal pattern (see inset of Fig. 1). The doubling pattern observed in Fig. 1 is also revealed by the time evolution of the central density, which is given by $\rho_{c}(t) \equiv N|\Psi(0, t)|^{2}$, in Fig. 2. The observed strong fluctuations in the density increase for larger times. These results support a conjecture that the system, by doubling the peaks indefinitely, starts to excite a whole spectrum of frequencies.

One can also observe a striking feature in Fig. 1, that the number of atoms described by the NLSE go far beyond the static critical limit, in the long time scale. ${ }^{1}$ In a dynamical situation, one should note that the number of atoms can grow beyond $N_{c}$, which is not possible in the static case. $N_{c}$ refers to the critical limit for the number of atoms in the ground state; however, the excited states allow more atoms than the

\footnotetext{
${ }^{1}$ This behavior is not observed in the numerical solutions of the quantum Boltzmann depicted in Fig. 1 of Ref. [4]
} 


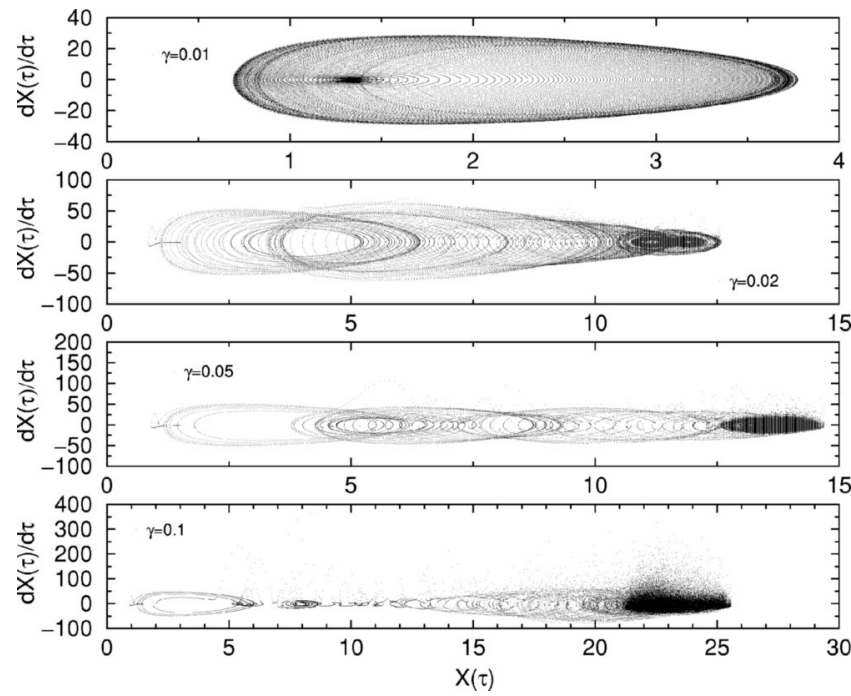

FIG. 3. Phase-space plots, characterized by different values of the feeding parameter $\gamma$ (given inside the plots), for the meansquare radius $X(\tau) \equiv \sqrt{\left\langle x^{2}(\tau)\right\rangle}$, in dimensionless units. In all the cases, $\xi$ equal to 0.001 and the wave functions were evolved until $\omega t=1000$.

ground state, as shown in Ref. [12]. So, the number of atoms higher than the static critical limit (after some period of time) can be interpreted as if not all the particles are in the ground state, but that the excited states are also becoming populated. After a long enough period of time ( $\omega t>100)$, one can observe that the number of atoms remains higher than $N_{c}$; a result which is consistent with the interpretation that a large fraction of the particles are populating the first radial excited state. Within the same interpretation, other excited states can be populated in a longer enough period of time. As a consequence, in the long time scale, the picture of a condensate with atoms in the ground state, assumed to be described by Eq. (1), is no longer valid, as the feeding process is populating the excited states. This scenario is also confirmed by the large values of the mean-square radius attained by the wave function, as shown in Fig. 3.

The present results show possible limitations of the model given in [3], when trying to describe BEC atoms with attractive two-body interactions. As verified in Fig. 3, the growth above the critical value is strongly dependent on the nonconservative parameters. One should also observe that in Ref. [3] they have considered the case of a decreasing time dependent $\gamma$, that shows saturation just after the beginning of the collapses. Effectively, this is a way one can try for a better description of the experimental data, together with a more general study of the parameters in the nonlinear terms of the NLSE.

One particular interesting observable, to further analyze the dynamical behavior of Eq. 1, is the mean-square radius. We define this observable in dimensionless units by $X(\tau)$ $\equiv \sqrt{\left\langle x^{2}(\tau)\right\rangle}$. In Fig. 3, we plot $(d / d \tau) X(\tau)$ as a function of $X(\tau)$, for a set of values of the parameter $\gamma(\gamma$ $=0.01,0.02,0.05,0.1)$. In all cases, the wave-functions were evoluted up to $\omega t=1000$ and the strength of the three-body dissipative interaction is kept fixed at $\xi=0.001$. In Fig. 3, we observe that a complex dynamical structure starts to appear as the value of the parameter $\gamma$ increases. For $\tau=0$, $\sqrt{\left\langle x^{2}(\tau)\right\rangle}$ is close to 1.52 and $(d / d \tau) \sqrt{\left\langle x^{2}(\tau)\right\rangle}$ is zero, in all the cases. Initially, for $\gamma=0.01$, the radius decreases to a center near 1.34 with zero derivative, then it starts to oscillate with larger radius, but keeping the center fixed. A similar behavior is found for $\gamma<0.01$. For larger values of $\gamma$, the center of the oscillation in $X$ grows up to the point it reaches an attractor at very large radius. In case of $\gamma=0.1$, for example, the plot clearly resembles a chaotic behavior with a strange attractor, which is around $X(\tau) \sim 23$. We have observed a fast transition in the pattern of the trajectory, when comparing the results obtained for $\gamma=0.01$ and $\gamma=0.012$, in a similar plot. This gives an indication of the existence of a critical range of values for the parameter $\gamma$ for the transition from order to chaos. In this model, the signature of the onset of chaos is a noncontinuous increasing of the radius up to very large values, compared to some typical ground-state value, as we found for $\gamma>0.02$. The chaotic behavior of the system, for this set of parameters, will be demonstrated through the calculation of the largest Lyapunov exponent, which will be defined in the following.

We should note that we kept fixed the dissipative term of Eq. (1); however, the indication of transition to chaos can also be found by changing $\xi$ with $\gamma$ fixed. In other words, by keeping fixed $\gamma$ to a certain nonzero value, a similar behavior is reached as we decrease the value of the parameter $\xi$. Our aim in the next is to determine the existence of spatiotemporal chaos in the time evolution of trapped atoms described by Eq. (1), by considering a general procedure for nonlinear partial differential equations.

In [5], it was studied the complex quintic GinzburgLandau equation and showed that, for an appropriate choice of the parameters the system could present a chaotic behavior. In order to characterize the chaotic behavior, for a spatiotemporal equation, the following function $\zeta$ was defined [5]:

$$
\zeta(\tau)=\left(\int_{0}^{L}|\delta \Phi(x, \tau)|^{2} d x\right)^{1 / 2}
$$

The average slope of this function plotted as a function of time, gives the largest Lyapunov exponent [5]. The chaotic behavior is characterized by a positive slope. The calculation of $\delta \Phi$ as described in Ref. [5] can also be related to the excitation of collective modes by an infinitesimal perturbation of the wave function. The onset of the chaotic behavior can be interpreted as the increase of the magnitude of the collective excitations, or alternatively, by the creation of many quasiparticle states, with the corresponding depletion of the ground-state occupation $[13,14]$. In this sense, the mean-field approximation should be valid for times in which the wavelength is much larger than the average interparticle distance.

The formal main difference between the complex quintic Ginzburg-Landau equation and the Eq. (1) is the presence of the trap in the latter. The similarity of these equations led us to consider the criterion used in Ref. [5], applying the Eq. (3) to the wave functions obtained from Eq. (1). We are mainly 


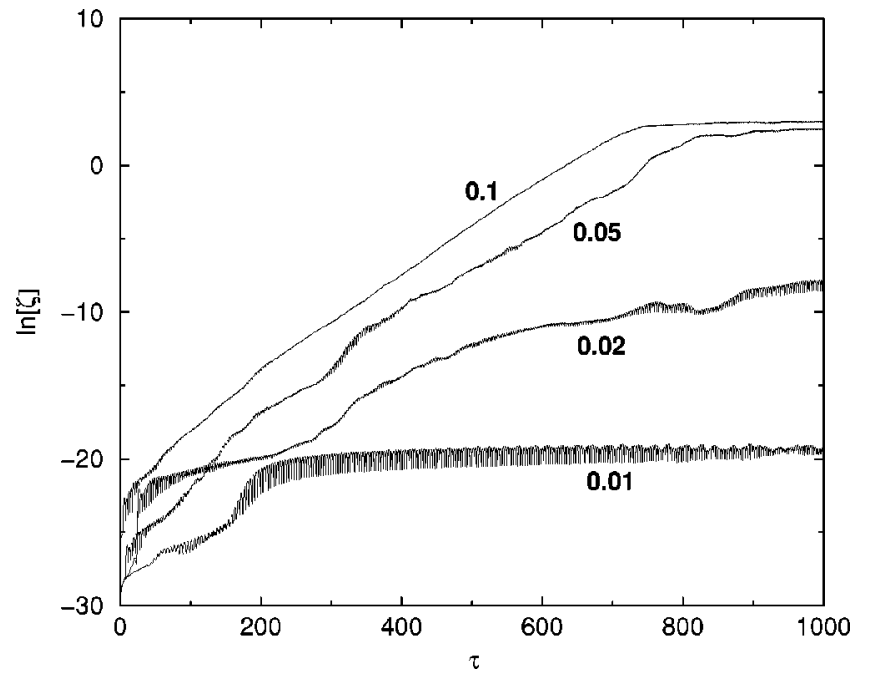

FIG. 4. Logarithmic representation of the separation between two nearby states, as given by Eq. (3), for several values of the parameter $\gamma$ (shown near the corresponding plot). $\xi$ is maintained fixed to 0.001 and $\tau \equiv \omega t$. All quantities are given in dimensionless units.

interested in studying the time evolution of the condensed wave function for a negative two-atom scattering length, through the investigation of the numerical accuracy of the results for certain parameters used. By considering a general example, we keep fixed the value of the three-body dissipative parameter $\xi$ ( $\xi=0.001$, as in Ref. [3]), and study a range of values for the feeding parameter.

In order to calculate Eq. (3), we use the same initial wave function $\Phi(x, \tau)$ as in Ref. [3], i.e., with initial number of atoms equal to $0.75 N_{c}$. This initial wave function was also evoluted with an added small random perturbation $\delta \Phi(x, 0)$ $\sim 10^{-14}$. The difference between the wave function and the perturbed wave function gives the separation of the trajectories $\delta \Phi(x, \tau)$, which is used to obtain our results for Eq. (3) shown in Fig. 4. As one can observe, there is an approximately exponential increase in $\zeta$ as the time grows for all cases presented with $\gamma>0.01$, such that we can draw a conclusion about the chaotic behavior of Eq. (1). This is better characterized for $\gamma=0.1$. This confirms the suspicion raised when analyzing the results obtained in Figs. 1-3, as one can clearly obtain from such results the values of the feeding parameter for the system to become chaotic.

In conclusion, the NLSE used for the description of the dynamics of the Bose condensed wave function in atomic traps with attractive interactions in [3], for certain class of parameters (as, for example, the parameters considered in [3], $\xi=0.001$ and $\gamma=0.1$ ), is chaotic. The chaotic behavior of Eq. (1) starts to disappear as one decreases $\gamma$ or increases $\xi$. In our understanding, this is an important result of the formalism considered for the description of Bose-Einstein condensed states. The onset of chaos is accompanied by a noncontinuous increasing of the radius up to very large values, compared to some typical ground-state value. As excited states start to be populated, the feeding process described by the equation is not limited to the ground state, and a chaotic regime is observed in the model under consideration. In this sense, the validity of the mean-field approximation to describe the physical system is questionable in the long-time domain. Further investigations are in progress.

We would like to thank Professors C. Grebogi, R.G. Hulet, and F.Kh. Abdullaev for useful and stimulating discussions. We also thank G.V. Shlyapnikov and A.E. Muryshev for useful help concerned with the numerical approach. This work was partially supported by Fundação de Amparo à Pesquisa do Estado de São Paulo and Conselho Nacional de Desenvolvimento Científico e Tecnológico.
[1] M. Edwards and K. Burnett, Phys. Rev. A 51, 1382 (1995); P.A. Ruprecht, M.J. Holland, K. Burnett, and M. Edwards, ibid. 51, 4704 (1995).

[2] C.C. Bradley, C.A. Sackett, and R.G. Hulet, Phys. Rev. Lett. 78, 985 (1997); C.C. Bradley, C.A. Sackett, J.J. Tollet, and R.G. Hulet, ibid. 79, 1170 (1997).

[3] Y. Kagan, A.E. Muryshev, and G.V. Shlyapnikov, Phys. Rev. Lett. 81, 933 (1998).

[4] C.A. Sackett, J.M. Gerton, M. Welling, and R.G. Hulet, Phys. Rev. Lett. 82, 876 (1999).

[5] R.J. Deissler and K. Kaneko, Phys. Lett. A 119, 397 (1987); R.J. Deissler, J. Stat. Phys. 54, 1459 (1989); H.R. Brand and R.J. Deissler, Phys. Rev. E 58, R4064 (1998).

[6] M.J. Ablowitz, C.M. Schober, and B.M. Herbst, Phys. Rev. Lett. 71, 2683 (1993); M.J. Ablowitz, B.M. Herbst, and C.M.
Schober, Physica A 228, 212 (1996).

[7] W. Krauth, Phys. Rev. Lett. 77, 3695 (1996).

[8] A. Gammal, T. Frederico, L. Tomio, and Ph. Chomaz, Phys. Rev. A 61, 051602(R) (2000).

[9] W.F. Ames, Numerical Methods for Partial Differential Equations, 3rd ed. (Academic Press, New York, 1992), pp. 111115.

[10] J.M. Gerton, C.A. Sackett, B.J. Frew, and R.G. Hulet, Phys. Rev. A 59, 1514 (1999).

[11] Y. Kagan, E.L. Surkov, and G.V. Shlyapnikov, Phys. Rev. Lett. 79, 2604 (1997).

[12] A. Gammal, T. Frederico, and L. Tomio, Phys. Rev. E 60, 2421 (1999).

[13] Y. Castin and R. Dum, Phys. Rev. Lett. 79, 3553 (1997).

[14] M. Fliesser and R. Graham, Physica D 131, 141 (1999). 